\title{
Musashi 1-positive cells derived from mouse embryonic stem cells treated with LY294002 are prone to differentiate into intestinal epithelial-like tissues
}

\author{
SHAO-YANG LAN ${ }^{1,2 *}$, MEI-AO TAN ${ }^{3 *}$, SHU-HUI YANG $^{3}$, JIA-ZHONG CAI $^{4}$, BIN CHEN $^{1}$, \\ PEI-WU LI ${ }^{1}$, DONG-MEI FAN ${ }^{1}$, FENG-BIN LIU ${ }^{1}$, TAO YU ${ }^{2}$ and QI-KUI CHEN ${ }^{2}$
}

\author{
${ }^{1}$ Department of Spleen and Stomach Diseases, The First Affiliated Hospital of Guangzhou University of Chinese Medicine, \\ Guangzhou, Guangdong 510405; ${ }^{2}$ Department of Gastroenterology, The Second Affiliated Hospital, \\ Sun Yat-Sen University, Guangzhou, Guangdong 510120; ${ }^{3}$ First Clinical Medical College, and ${ }^{4} \mathrm{Pi}-$ Wei Institute, \\ Guangzhou University of Chinese Medicine, Guangzhou, Guangdong 510405, P.R. China
}

Received December 13, 2017; Accepted March 13, 2019

DOI: $10.3892 /$ ijmm.2019.4145

\begin{abstract}
The majority of Musashi 1 (Msi1)-positive cells derived from mouse embryonic stem cells (mESCs) are prone to differentiate into neural epithelial-like cells, and only a small proportion of Msi1-positive cells differentiate into intestinal epithelial-like cells. Whether inhibiting the phosphatidylinositol 3-kinase (PI3K) signaling of mESCs can promote the differentiation of Msi1-positive cells into intestinal epithelial-like cells remains to be fully elucidated. In the present study, to inhibit PI3K signaling, mESCs were treated with LY294002. A pMsi1-green fluorescence protein reporter plasmid was used to sort the Msi1-positive cells from mESCs treated and untreated with LY294002 (5 $\mu \mathrm{mol} / \mathrm{l})$. The Msi1-positive cells were hypodermically engrafted into the backs of non-obese diabetic/severe combined immunodeficient
\end{abstract}

Correspondence to: Mr. Qi-Kui Chen or Mr. Tao Yu, Department of Gastroenterology, The Second Affiliated Hospital, Sun Yat-Sen University, 107 Yan Jiang Xi Road, Guangzhou, Guangdong 510120, P.R. China

E-mail: qkchen2015@163.com

E-mail: yutao2014@126.com

*Contributed equally

Abbreviations: Msi1, Musashi 1; mESCs, mouse embryonic stem cells; NOD/SCID, non-obese diabetic/severe combined immunodeficient; GFP, green fluorescent protein; FACS, fluorescence-activated cell sorting; PI3K, phosphatidylinositol 3-kinase; Lgr5, leucine-rich repeat-containing G-protein coupled receptor; GSK3 $\beta$, glycogen synthase kinase-3 $\beta$; DMSO, dimethyl sulfoxide; LIF, leukemia inhibitory factor; CCK-8, Cell Counting Kit-8; Pdgf- $\alpha$, platelet-derived growth factor receptor $\alpha$; qPCR, quantitative polymerase chain reaction

Key words: intestinal epithelial stem cells, neural epithelial stem cells, phosphatidylinositol 3-kinase signaling, Musashi 1, embryonic stem cell mice. The presence of neural and intestinal epithelial-like cells in the grafts was detected by reverse transcription-quantitative polymerase chain reaction analysis and immunohistochemistry. Compared with the Msil-positive cells derived from mESCs without LY294002 treatment, Msi1-positive cells derived from mESCs treated with LY294002 expressed higher levels of leucine-rich repeat-containing G-protein coupled receptor, a marker of intestinal epithelial stem cells, and lower levels of Nestin, a marker of neural epithelial stem cells. The grafts from Msi1-positive cells treated with LY294002 contained more intestinal epithelial-like tissues and fewer neural epithelial-like tissues, compared with those from untreated Msi1-positive cells. LY294002 had the ability to promote the differentiation of mESCs into intestinal epithelial-like tissues. The Msil-positive cells selected from the cell population derived from mESCs treated with LY294002 exhibited more characteristics of intestinal epithelial stem cells than those from the untreated group.

\section{Introduction}

Musashi1 (Msi1) is an RNA-binding protein that is expressed at high levels in the nervous system $(1,2)$. It has been suggested that Msil serves an important role in maintaining stem cell state, differentiation and tumorigenesis (3-7). Numerous studies have shown that Msi1 is expressed at high levels in the nervous system and the adult intestinal epithelial stem cell population. Msi1 is located in a small number of epithelial cells directly above Paneth cells present in the small intestine crypts of adult mice. This indicates that Msi1 is a marker of intestinal epithelial stem cells $(8,9)$. Msi1-positive cells are regarded as a candidate for intestinal epithelial stem cells. As Msi1 protein is localized in the cytoplasm and nucleus of cells, and Msi1-positive cells are rarely distributed in the small intestinal crypt, it is almost impossible to harvest living Msi1-positive cells from the small intestine $(10,11)$. The scarcity of Msi1-positive cells has hindered investigation of the association between Msi1 and intestinal epithelial stem cells. 
Embryonic stem cells (ESCs) can differentiate into various types of cells, including Msi1-positive cells $(12,13)$. As Msi1-positive cells are a potential candidate for intestinal epithelial stem cells, Msi1-positive cells originated from ESCs are expected to develop into intestinal epithelial cells. It has also been demonstrated that Msi1 is selectively expressed in neural progenitor cells $(6,14,15)$. Therefore, Msi1 is considered a marker of neuroepithelial stem cells, and Msi1-positive cells from ESCs maintain their ability to differentiate into neuroepithelial tissue.

Living Msi1-positive cells cannot be selected by binding to surface antibodies due to the Msi1 protein being present in the interior of the cell. In our previous study, a reporter gene plasmid comprising a green fluorescent protein (GFP) sequence, pMsi1-GFP, was constructed under the control of the Msi1 promoter (16). Following transfection with the pMsi1-GFP vector, Msi1-positive cells were inspected in real time and effectively selected from a cell population derived from ESCs using flow cytometry (FCM). The selected Msi1-positive cells were able to differentiate into neural and intestinal epithelial-like cells in vivo. The majority of Msi1-positive cells derived from ESCs were prone to differentiate into neural epithelial-like cells, and only a small number of Msi1-positive cells differentiated into intestinal epithelial-like cells. This indicated that the selected Msi1-positive cells were similar to neural epithelial stem cells and not intestinal epithelial stem cells. Screening for Msi1-positive cells that tend to differentiate into intestinal epithelial stem cells is a prerequisite for investigating intestinal epithelial stem cells.

During embryogenesis, intestinal epithelial tissue develops from endoderm, and neural epithelial tissue develops from ectoderm (17-20). Therefore, it was reasonable to presume that the promotion of ESC differentiation into endoderm can increase the production of intestinal epithelial tissue. Phosphatidylinositol 3-kinase (PI3K) is extensively expressed and is core in monitoring numerous cellular processes, including cell migration, activation, differentiation, apoptosis and angiogenesis (21-23). It has been reported that PI3K signaling is involved in a broad array of elementary cellular responses and serves a critical role in the differentiation of ESCs (24-26). The suppression of PI3K signaling promotes the differentiation of ESCs into mesendoderm and inhibits their differentiation into ectoderm (27). Once ESCs differentiate into mesendoderm cells, Msi1-positive cells differentiate into intestinal epithelial stem cells, rather than neural stem cells. Msi1-positive cells sorted from mesendoderm cells are more favorable for the investigation of intestinal epithelial stem cells. LY294002 (2-4-morpholinyl-8-phenlchromone), a chemical inhibitor of PI3K, has been extensively used to examine the role of the PI3K signaling pathway $(28,29)$. The present study aimed tried to determine whether suppressing PI3K signaling can promote the differentiation of ESCs into Msi1-positive cells, which are prone to develop into intestinal epithelial-like tissue.

\section{Materials and methods}

Reagent preparation. LY294002 (Beyotime Institute of Biotechnology, Shanghai, China) was dissolved in dimethyl sulfoxide (DMSO) at a concentration of $10 \mathrm{mg} / \mathrm{ml}$ and stored at $-20^{\circ} \mathrm{C}$. The concentrations of LY294002 solutions finally used were $1.25,2.5,5,10,20$ and $50 \mu \mathrm{mol} / 1$.

Culture of mouse (m)ESCs and cell cycle analysis. The ES-E14TG2a mESCs were supplied by the American Type Culture Collection (Manassas, VA, USA). The undifferentiated mESCs were cultivated on gelatin-coated dishes without feeder cells in Dulbecco's modified Eagle's medium (DMEM; Gibco; Thermo Fisher Scientific, Inc., Waltham, MA, USA), supplemented with $10 \%$ fetal calf serum (FCS; HyClone; GE Healthcare Life Sciences, Logan, UT, USA), 10 mM HEPES (Gibco; Thermo Fisher Scientific, Inc.), 0.12\% sodium bicarbonate, $0.1 \mathrm{mM}$ nonessential amino acids (HyClone), $0.1 \mathrm{mM}$ 2-mercaptoethanol (2ME; Gibco; Thermo Fisher Scientific, Inc.), $100 \mathrm{U} / \mathrm{ml}$ penicillin $\mathrm{G}, 100 \mu \mathrm{g} / \mathrm{ml}$ streptomycin and $1,000 \mathrm{U} / \mathrm{ml}$ leukemia inhibitory factor (LIF; Chemicon International; Thermo Fisher Scientific, Inc.). The cells were cultured in a humidified $37^{\circ} \mathrm{C}$ incubator in a $5 \% \mathrm{CO}_{2}$-air mixture.

The proliferation rate of mESCs was measured with Cell Counting Kit-8 (CCK-8) (Dojindo Molecular Technologies, Kumamoto, Japan). Briefly, the mESCs were treated with trypsin $(0.25 \%)$ /EDTA $(0.02 \%)$ and $5 \times 10^{3}$ cells $(100 \mu \mathrm{l}$ volume/well) were plated in triplicate in gelatin-coated wells of a 96-well plate in media containing $1,000 \mathrm{U} / \mathrm{ml} \mathrm{LIF}$. LY294002 $(1.25,2.5,5,10,20$ and $50 \mu \mathrm{mol} / \mathrm{l})$ was mixed in triplicate wells and cultured at $37^{\circ} \mathrm{C}$ for $24 \mathrm{~h}$. DMSO was used as a vehicle control. Following treatment, $10 \mu \mathrm{l}$ of CCK-8 was added to each microculture well, and the absorbance at $450 \mathrm{~nm}$ was measured with a microplate reader following incubation for $2 \mathrm{~h}$ at $37^{\circ} \mathrm{C}$.

Differentiation of $m E S C s$ and formation of embryonic bodies $(E B s)$. To induce mESC differentiation, LIF was removed from the mESC culture medium (EB medium). The mESCs were cultured using the hanging-drop method ( $32 \mu \mathrm{l}$ per drop) to form EBs at a concentration of $1 \times 10^{6}$ cells $/ \mathrm{ml}$ in EB medium with or without LY294002. After 2 days, the harvested EBs were dissociated with trypsin (0.25\%)/EDTA (0.02\%). They were inoculated at a concentration of $1 \times 10^{5}$ cells/well in a 6-well culture plate (Nalge Nunc International; Thermo Fisher Scientific, Inc.) and cultured in EB medium with or without LY294002. All EB cell cultures were maintained in a humidified chamber in a $5 \% \mathrm{CO}_{2}$-air mixture at $37^{\circ} \mathrm{C}$.

Western blot analysis. Whole-cell lysates were prepared using RIPA lysis buffer (Beyotime Institute of Biotechnology) and centrifuged $(10,000 \mathrm{x} \mathrm{g})$ at $4^{\circ} \mathrm{C}$ for $15 \mathrm{~min}$. Total protein in the supernatant of the cell lysate was measured by the BCA Protein Assay kit (Beyotime Institute of Biotechnology). The supernatants were boiled for $5 \mathrm{~min}$ and were size-fractionated by sodium dodecyl sulfate-polyacrylamide gel electrophoresis (12\% acrylamide; $40 \mu \mathrm{g}$ protein per sample). The proteins were transferred onto nitrocellulose filters, following which the blots were incubated with rabbit anti-mouse glycogen synthase kinase-3 $\beta$ (GSK3 $\beta$; cat. no. NBP1-31353), rabbit anti-mouse phosphorylated GSK3 $\beta^{\mathrm{S9}}$ (cat. no. AF1590-SP; both 1:500; Novus Biologicals, Littleton, CO, USA) and rabbit anti-mouse $\beta$-actin (1:2,000; cat. no. 4970; Cell Signaling Technology, Inc., Danvers, MA, USA) antibodies overnight 
at $4^{\circ} \mathrm{C}$. The secondary antibodies used were horseradish peroxidase-conjugated anti-rabbit (1:4,000; cat. no. 7074; Cell Signaling Technology, Inc.), which were incubated for $1 \mathrm{~h}$ at room temperature with gentle agitation. GSK3 $\beta$ and GSK $3 \beta^{\mathrm{S} 9}$ were detected using an ECL chemiluminescence system (Pierce; Thermo Fisher Scientific, Inc.). The integrated intensity for the protein bands was determined by scanning densitometry and analyzed via Glyko BandScan 5.0 (Glyko, Novato, CA, USA).

Construction of pMsil-GFP and cell transfection. A reporter gene plasmid (pMsi1-GFP) was constructed in our previous study, and Msi1-positive cells were selected from a cell population derived from mESCs by fluorescence-activated cell sorting (FACS). The day before transfection, $2 \times 10^{5}$ of the 9-day cell population derived from mESCs were counted and seeded into 6-well plates in EB medium with or without LY294002. For generation of a Lipofectamine-plasmid DNA complex, $150 \mu \mathrm{l}$ of serum-free medium was mixed with $10 \mu \mathrm{l}$ of Lipofectamine (Invitrogen; Thermo Fisher Scientific, Inc.) and incubated for $5 \mathrm{~min}$ at room temperature. Subsequently, $150 \mu \mathrm{l}$ of DMEM was mixed with $4 \mu \mathrm{g}$ of pMsi1-GFP, added to Lipofectamine solution, mixed gently and incubated for $30 \mathrm{~min}$ at room temperature. The transfection complexes were then added to each well. The medium was replaced following $6 \mathrm{~h}$ of incubation with EB culture medium with or without LY294002. The GFP-positive cells were counted using FCM (Beckman Coulter, Inc., Brea, CA, USA) $24 \mathrm{~h}$ following transient transfection. The cells were counterstained with Hoechst 33342 (10 $\mu \mathrm{g} / \mathrm{ml}$; Sigma-Aldrich; Merck KGaA, Darmstadt, Germany) at room temperature for $10 \mathrm{~min}$ and observed under a fluorescence microscope (Olympus iX71; Olympus Corporation, Tokyo, Japan).

FACS and grafting. The cells were harvested using trypsin $(0.25 \%) /$ EDTA $(0.02 \%) 24 \mathrm{~h}$ following transfection. The LY-Msi1-positive cells and EB-Msil positive cells were selected from 10-day cell populations treated with and without LY294002, respectively, using FCM (488 nm), gated for a high expression level of GFP. The selected LY/EB-Msil positive cells were harvested $\left(\sim 5.0 \times 10^{5}\right.$ cells per dose $)$ whereas the unselected 10-day cell populations (treated with or without LY294002) were injected subcutaneously into 20 non-obese diabetic/severe combined immunodeficient (NOD/SCID) mice (female, 4-6-week-old and 14-20 g; $n=5$ in each group; Laboratory Animal Center of Sun Yat-Sen University, Guangzhou, China). Throughout the experiment, the mice were housed in a room with a 12/12-h light/dark cycle, at a temperature of $27^{\circ} \mathrm{C}$, with $60 \%$ relative humidity and free access to chow and water. At 2 weeks post transplantation, the mice were sacrificed and the grafts were removed. All experiments were performed in triplicate and repeated separately three times. All procedures were performed in accordance with the Animal Ethics Committee of the Second Affiliated Hospital of Sun Yat-Sen University.

Reverse transcription-quantitative polymerase chain reaction (RT-qPCR) analysis. Total RNA from the cells and grafts were extracted using TRIzol reagent (Invitrogen; Thermo Fisher Scientific, Inc.). The concentration and purity of the total RNA were detected using a UV-2450 spectrophotometer (Shimadzu Corporation, Kyoto, Japan). According to the manufacturer's protocol, $1 \mu \mathrm{g}$ of total RNA was used to perform cDNA synthesis, using a ReverTra Ace- $\alpha-{ }^{\circledR}$ kit (Toyobo Life Science, Osaka, Japan). qPCR analysis was performed using a Real-time ${ }^{\mathrm{TM}}$ PCR Master Mix kit (Toyobo Life Science) and SYBR Premix Ex Taq ${ }^{\mathrm{TM}}$ II (Takara Bio, Inc., Otsu, Japan) in a LightCycler 480 (Roche Diagnostics, Basel, Switzerland). The cycling conditions were $95^{\circ} \mathrm{C}$ for $30 \mathrm{sec}$, followed by 40 cycles of $95^{\circ} \mathrm{C}$ for $15 \mathrm{sec}$ and $60^{\circ} \mathrm{C}$ for $30 \mathrm{sec}$. The experiment was repeated twice and the results were averaged. Data were analyzed using the $\Delta \Delta \mathrm{Ct}$ method with $\beta$-actin as the constitutive marker (30).

The sequences of the primers used were as follows (and reverse): Mouse Msi1, forward 5'-ATGGTGGAATGCAAG AAAGC-3' and reverse 5'-TAGGTGTAACCAGGGGC AAG-3'; $\beta$-actin, forward 5'-CGGCTACCACATCCAAG GAA-3' and reverse 5'-GCTGGAATTACCGCGGCT-3'; mouse Tubulin $\beta$ III, forward 5'-CTTCGGGCAGATCTT CAGAC-3' and reverse 5'-AGTCAACCAGCTCTGCA CCT-3'; mouse Villin forward 5'-ACGGTGGTGACTGCT ACCTGCT-3' and reverse 5'-AACCACCATGCGGCCCT TGA-3'; mouse platelet-derived growth factor receptor $\alpha$ (Pdgfr- $\alpha$ ), forward 5'-AACCTTCAGCGTGGGGCCTT-3' and reverse 5'-ACAGTCTGGCGTGCGTCCAT-3'; mouse Leucine-rich repeat-containing G-protein coupled receptor 5 (Lgr5), forward 5'-CACCAGCTTACCCCATGACT-3' and reverse 5'-CTCCTGCTCTAAGGCACCAC-3'; mouse Nestin, forward 5'-GAGAAGACAGTGAGGCAGATGAGTTA-3' and reverse 5'-GCCTCTGTTCTCCAGCTTGCT-3.

Immunohistochemistry. Immunohistochemical analysis was performed to measure the protein expression of Tubulin $\beta$ III and Villin. The grafts were removed from the NOD/SCID mice and fixed with $4 \%$ paraformaldehyde overnight at $4^{\circ} \mathrm{C}$, embedded in paraffin, and cut at a thickness of $6 \mu \mathrm{m}$. The sections were washed for $15 \mathrm{~min}$ in PBS and subjected to antigen retrieval using a microwave oven for $15 \mathrm{~min}$. The sections were cooled to room temperature and incubated for $2 \mathrm{~h}$ with $10 \%$ normal goat (or rabbit) serum (cat. no. kit-9710; Ultersensitive SP kit; Fuzhou Maixin Biotech Co., Ltd., Fuzhou, China) to reduce nonspecific binding. After a 15 min PBS wash, the sections were incubated overnight at $4^{\circ} \mathrm{C}$ with rabbit anti-mouse Tubulin $\beta$ III $(2.5 \mathrm{mg} / \mathrm{ml}$ in PBS; cat. no. 1967-1, Epitomics; Abcam, Cambridge, MA, USA), or goat anti-mouse Villin $(5 \mathrm{mg} / \mathrm{ml}$ in PBS; cat. no. Sc-7672, Santa Cruz Biotechnology, Inc., Dallas, TX, USA) antibodies. After a 15 min PBS wash, the sections were treated with biotinylated anti-rabbit (or goat) IgG antibody (1:200 dilution; Ultersensitive SP kit) at $37^{\circ} \mathrm{C}$ for $60 \mathrm{~min}$. A positive reaction was detected using the SP method (Ultersensitive SP kit) and visualized via a diaminobenzidine reaction (Ultersensitive SP kit). The sections were counterstained with hematoxylin (cat. no. CTS-1099; Maixin Biotech Co., Ltd.) at room temperature for $30 \mathrm{sec}$, prior to being imaged under a iX71 fluorescence microscope.

Statistical analysis. Statistical analyses were performed using a statistical software package (SAS 8 for Windows; SAS Institute, Inc., Cary, NC, USA). Data are presented as 
the mean \pm standard derivation. Data were analyzed using one-way analysis of variance and $\alpha=0.05(\mathrm{P}<0.05)$ was used to indicate a statistically significant difference between separate groups.

\section{Results}

Effects of LY294002 in vitro: Suppression of cell survival. As an inhibitor of PI3K signaling, LY294002 suppresses the proliferation of mESCs (31). To determine the appropriate concentration of LY294002 in the present study, the mESCs were treated with LY294002 at a concentration gradient for $24 \mathrm{~h}$. When the mESCs were treated with $1.25,2.5,5,10$, 20 and $50 \mu \mathrm{mol} / 1 \mathrm{LY} 294002$, the mean absorbance was $73.2 \pm 4.5,68.0 \pm 3.4,62.0 \pm 2.2,39.5 \pm 1.7,4.8 \pm 1.7$ and $0.8 \pm 0.4 \%$, respectively, showing a decreasing trend. When DMSO was used as a vehicle in the cell culture, the mean absorbance was $97.5 \pm 2.3,89.3 \pm 6.8,89.4 \pm 4$., $91.1 \pm 11.9,95.2 \pm 2.0$ and $83.2 \pm 1.2 \%$, respectively. As shown in Fig. 1, the proliferation of mESCs was inhibited by LY294002 in a dose-dependent manner. The inhibitory effect was significant at a concentration of $>5 \mu \mathrm{mol} / 1$. Therefore, $5 \mu \mathrm{mol} / 1 \mathrm{LY} 294002$ was subsequently used.

Effects of LY294002 in vitro: Inactivation of GSK3 $\beta$ in mESCs. The suppression of PI3K signaling by LY294002 was affirmed by probing the cell lysates with phosphospecific antibodies that discerned the activity status of downstream PI3K signaling effectors, including GSK3 $\beta$. These outcomes demonstrated that $5 \mu \mathrm{mol} / 1 \mathrm{LY} 294002$ inhibited PI3K signaling within $72 \mathrm{~h}$, as shown by a collapse in the phosphorylation of GSK $3 \beta^{\mathrm{Sg}}$ (Fig. 2A and B). It was concluded that $5 \mu \mathrm{mol} / 1 \mathrm{LY} 294002$ was sufficient to inhibit the canonical PI3K signaling pathway, but had limited effect on the proliferation of mESCs.

Effect of LY294002 on the mRNA expression of Msil during the differentiation of $m E S C s$. The mRNA expression levels of Msil were gradually increased during the differentiation of mESCs treated with or without $5 \mu \mathrm{mol} / 1 \mathrm{LY} 294002$. Compared with the mESCs, the mRNA expression levels of Msi1 in the 8-13-day cell populations derived from mESCs without LY294002 treatment were $1.103 \pm 0.060,1.460 \pm 0.120$, $4.090 \pm 0.190,5.543 \pm 0.190,3.867 \pm 0.159$ and $3.513 \pm 0.170$, respectively. Those treated with LY94002 were $0.977 \pm 0.048$, $1.2780 \pm 0.111,4.667 \pm 0.234,3.967 \pm 0.264,2.730 \pm 0.210$ and $1.502 \pm 0.167$, respectively (Fig. 3). Taken together, the mRNA expression levels of Msil for cell populations derived from mESCs without LY294002 treatment were higher than those of the treated group at each time point, with the exception of the 10-day cell population $(\mathrm{P}<0.05)$. The mRNA expression level of Msi1 in the 10-day cell population derived from mESCs treated with LY294002 was higher than that of the untreated group $(\mathrm{P}<0.05)$.

Transient transfection of pMsil-GFP. At $24 \mathrm{~h}$ post-transfection with pMsi1-GFP, GFP-positive cells were found in the 10-day cell populations with or without LY294002 treatment (Fig. 4A-F). The positive expression rate of GFP was estimated with FCM. Following transfection with pMsi1-GFP, the positive expression rates of GFP in the 10-day cell

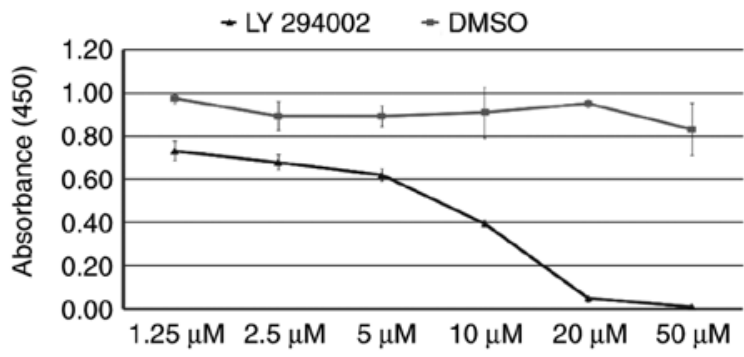

Figure 1. Influence of different concentrations of LY294002 on the proliferation of mESCs. LY294002 inhibited the proliferation of mESCs in a dose-dependent manner. The proliferation of mESCs was inhibited when the concentration of LY294002 was $>5 \mu \mathrm{mol} / 1$ and was completely inhibited when the concentration of LY294002 was $>20 \mu \mathrm{mol} / 1$. The concentration of DMSO used had limited influence on the proliferation of mESCs. mESCs, mouse embryonic stem cells; DMSO, dimethyl sulfoxide.

populations derived from mESCs with or without LY294002 treatment were $16.540 \pm 2.760$ and $10.040 \pm 1.980 \%$, respectively (Fig. 4G-I).

mRNA expression of markers for intestinal and neural epithelial stem cells in selected Msil-positive cells. The EB-Msil positive cells and LY-Msi1 positive cells were selected from the 10-day cell populations treated with or without LY294002. Compared with the 10-day cell population without LY294002 treatment, the mRNA expression levels of Msi1 in the EB-Msil positive cells, LY-Msil-positive cells and 10-day cell population treated with LY294002 were $12.731 \pm 2.145,11.864 \pm 2.231$ and $1.137 \pm 0.029$, respectively (Fig. 5). The mRNA expression levels of Msi1 in both Msi1-positive cell groups were higher than those in unselected cells treated with or without LY294002 $(\mathrm{P}<0.05)$. The mRNA expression level of Msi1 in EB-Msi1-positive cells was similar to that of LY-Msi1-positive cells ( $\mathrm{P}>0.05)$.

To determine whether Msi1-expressing cells were able to further differentiate into neural and intestinal epithelial cells, the mRNA expression levels of the markers for neural and intestinal epithelial stem cells were analyzed. Lgr5 has been reported as a marker of intestinal epithelial stem cells (32). Compared with the 10-day cell population without LY294002, the mRNA expression levels of Lgr5 in EB-Msi1-positive cells, LY-Msi1-positive cells and the 10-day cell population treated with LY294002 were $4.379 \pm 0.532,7.665 \pm 0.844$ and $4.616 \pm 0.665$, respectively (Fig. 5). The mRNA expression level of Lgr5 in the LY-Msil-positive cells was higher than those in the other groups $(\mathrm{P}<0.05)$. The mRNA expression levels of Lgr5 did not differ significantly between the EB-Msi1-positive cells and the 10-day cell population treated with LY294002 (P>0.05).

Nestin has been confirmed as a target of neural epithelial progenitors. Compared with the 10-day cell population without LY294002 treatment, the mRNA expression levels of Nestin in the EB-Msi1-positive cells, LY-Msi1-positive cells and the 10-day cell population treated with LY294002 were $5.112 \pm 0.679,0.377 \pm 0.032$ and $0.423 \pm 0.057$, respectively (Fig. 5). The mRNA expression level of Nestin in the EB-Msil-positive cells was higher than those in the other groups $(\mathrm{P}<0.05)$. The mRNA expression level of Nestin in the LY-Msi1-positive cells was similar to that in the 10-day cell 
A

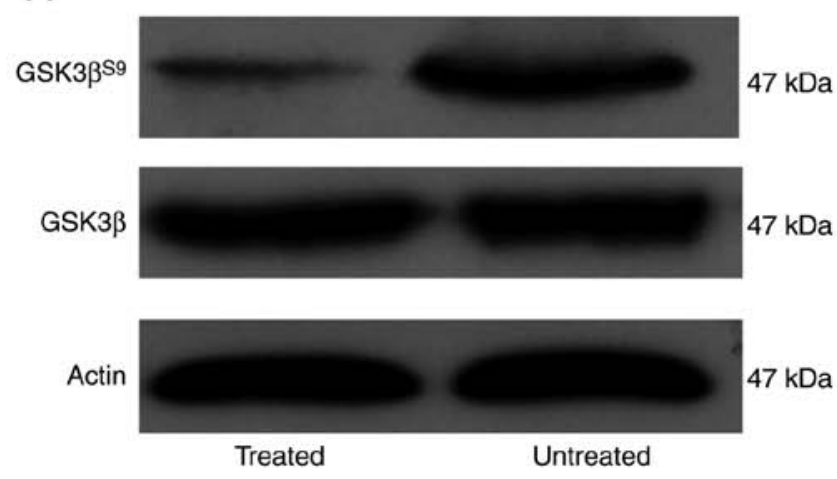

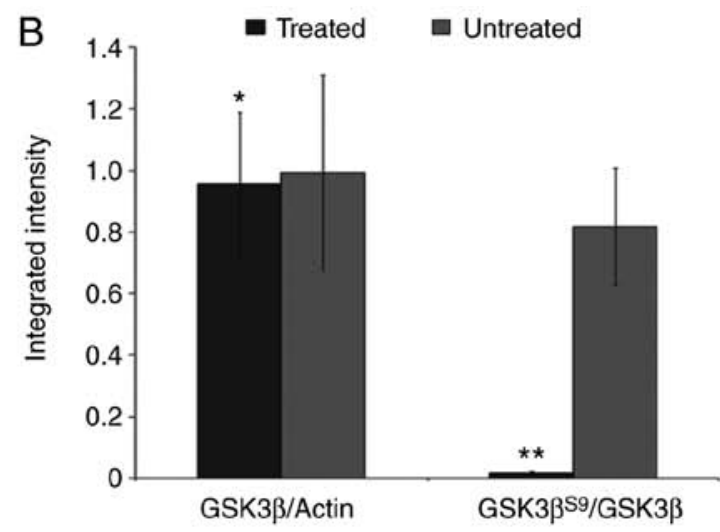

Figure 2. Western blotting showing LY294002 has an inhibitory effect on the phosphorylation of GSK3 $\beta$. mESCs were cultured in embryonic body culture medium treated with or without $5 \mu \mathrm{mol} / 1 \mathrm{LY} 294002$ for $72 \mathrm{~h}$. (A) Changes in GSK3 $\beta$ protein activity in response to LY294002 treatment (72 h) were determined using phosphospecific antibodies in immunoblot assays. An antibody recognizing pan-GSK $3 \beta$ was used to evaluate relative protein loading. The phosphorylation of GSK3 $\beta$ on Serine9 is shown in the top panel (GSK3 $\beta S 9$ ), the total level of GSK3 $\beta$ was detected in the middle panel, $\beta$-actin served as an internal control. (B) Protein expression levels of GSK3 $\beta$, GSK3 $\beta S 9$ and $\beta$-actin were indicated by the intensity of the corresponding bands. There was no difference between the integrated intensity ratio of GSK3 $\beta / \beta$-actin in mESCs treated with LY294002 (0.957 \pm 0.233$)$ or mESCs without LY294002 (0.994 \pm 0.317 ). The integrated intensity ratio of GSK3 $\beta S 9 / G S K 3 \beta$ in mESCs treated with LY294002 was $0.018 \pm 0.005$, which was lower than that in mESCs

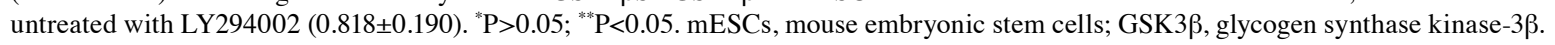

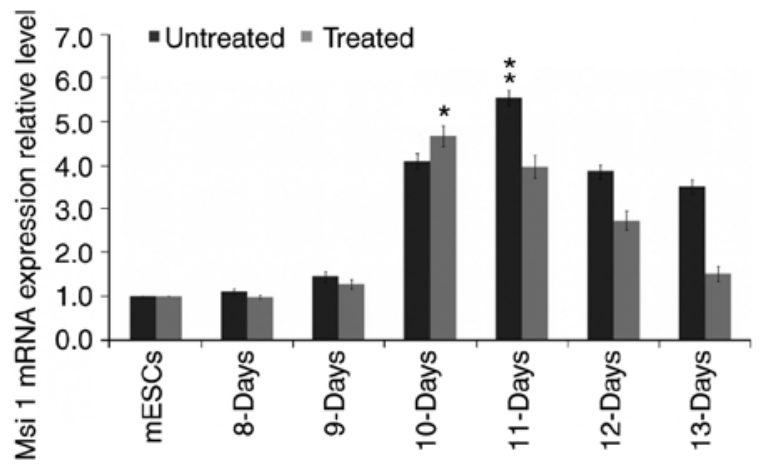

Figure 3. Relative mRNA expression of Msi1 in mESCs treated or untreated with LY294002. The mRNA expression of Msi1 reached a peak level in the 10-day cell population derived from mESCs treated with LY294002, whereas the mRNA expression of Msi1 reached a peak level in the 11-day cell population derived from mESCs without LY294002 treatment. The mRNA expression level of Msil in the 10-day cell population derived from mESCs treated with LY294002 was higher than that of cell populations derived from mESCs without LY294002 at each time point, but lower than that of the 11-day cell population derived from mESCs without LY294002. ${ }^{*} \mathrm{P}<0.05$; ${ }^{* *} \mathrm{P}<0.05$. Msi1, Musashi 1; mESCs, mouse embryonic stem cells.

population treated with LY294002 (P>0.05), and both were significantly lower than that in the 10-day cell population without LY294002 treatment $(\mathrm{P}<0.05)$.

Sorting of Msil-positive cells and grafting assay. To observe how the Msi1-positive cells differentiate in vivo, the present study separated and then engrafted LY/EB-Msil positive cells, in addition to the unselected 10-day cell populations treated with and without LY294002 following pMsi1-GFP transfection, into the backs of 5-week-old NOD/SCID mice. At 10 days post-injection, the grafts were developed. They grew quickly, and their diameters had reached $1-2 \mathrm{~cm}$ at 2 weeks after injection.

Tubulin $\beta$ III has been confirmed as a marker of mature neural epithelial cells (33-35). The mRNA expression levels of the Tubulin $\beta$ III in grafts from LY-Msi1-positive cells, EB-Msi1-positive cells and the 10-day cell population treated with LY294002 were 0.623 $\pm 0.073,2.08 \pm 0.318$ and $0.567 \pm 0.105$, respectively, compared with the 10 -day cell population without LY294002 (Fig. 6). The mRNA expression level of Tubulin $\beta$ III in the grafts from EB-Msil-positive cells was higher than those in the other groups $(\mathrm{P}<0.05)$. The mRNA expression levels of the Tubulin $\beta$ III in the grafts from the LY-Msi1-positive cells and the 10-day cell population treated with LY294002 were significantly lower than those in the grafts from EB-Msi1-positive cells and the 10-day cell population without LY294002 $(\mathrm{P}<0.05)$. These results indicated that EB-Msil-positive cells were prone to develop into neural epithelial-like tissue in vivo, compared with LY-Msi1-positive cells. Therefore, LY294002 suppressed the ability of mESCs to differentiate into neural epithelial-like tissue.

Villin, an actin bundling protein, is a dominant structural component of the brush border of intestinal absorptive cells $(36,37)$. The mRNA expression levels of Villin in grafts from the LY-Msi1-positive cells, EB-Msi1-positive cells and the 10-day cell population treated with LY294002 were $14.612 \pm 1.053,3.993 \pm 0.649$ and $4.422 \pm 1.233$, respectively, compared with that in the 10-day cell population without LY294002 (Fig. 6). The mRNA expression level of Villin in the grafts from LY-Msi1-positive cells was higher than those in other groups $(\mathrm{P}<0.05)$. The mRNA expression levels of the Villin in the grafts from EB-Msi1-positive cells and the 10-day cell population treated with LY294002 were higher than that in the grafts from the 10-day cell population without LY294002 $(\mathrm{P}<0.05)$. These results showed that LY294002 promoted the differentiation of mESCs into intestinal epithelial-like cells. LY-Msi1-positive cells were prone to develop into intestinal epithelial-like tissue in vivo.

Pdgfr- $\alpha$ is a typical mesoderm marker (38-40). The mRNA expression levels of Pdgfr- $\alpha$ in grafts from the LY-Msi1-positive cells, the 10-day cell population treated with LY294002 and EB-Msi1-positive cells were 0.094 \pm 0.005 , $1.893 \pm 0.294$ and $0.031 \pm 0.004$-fold, respectively, compared 

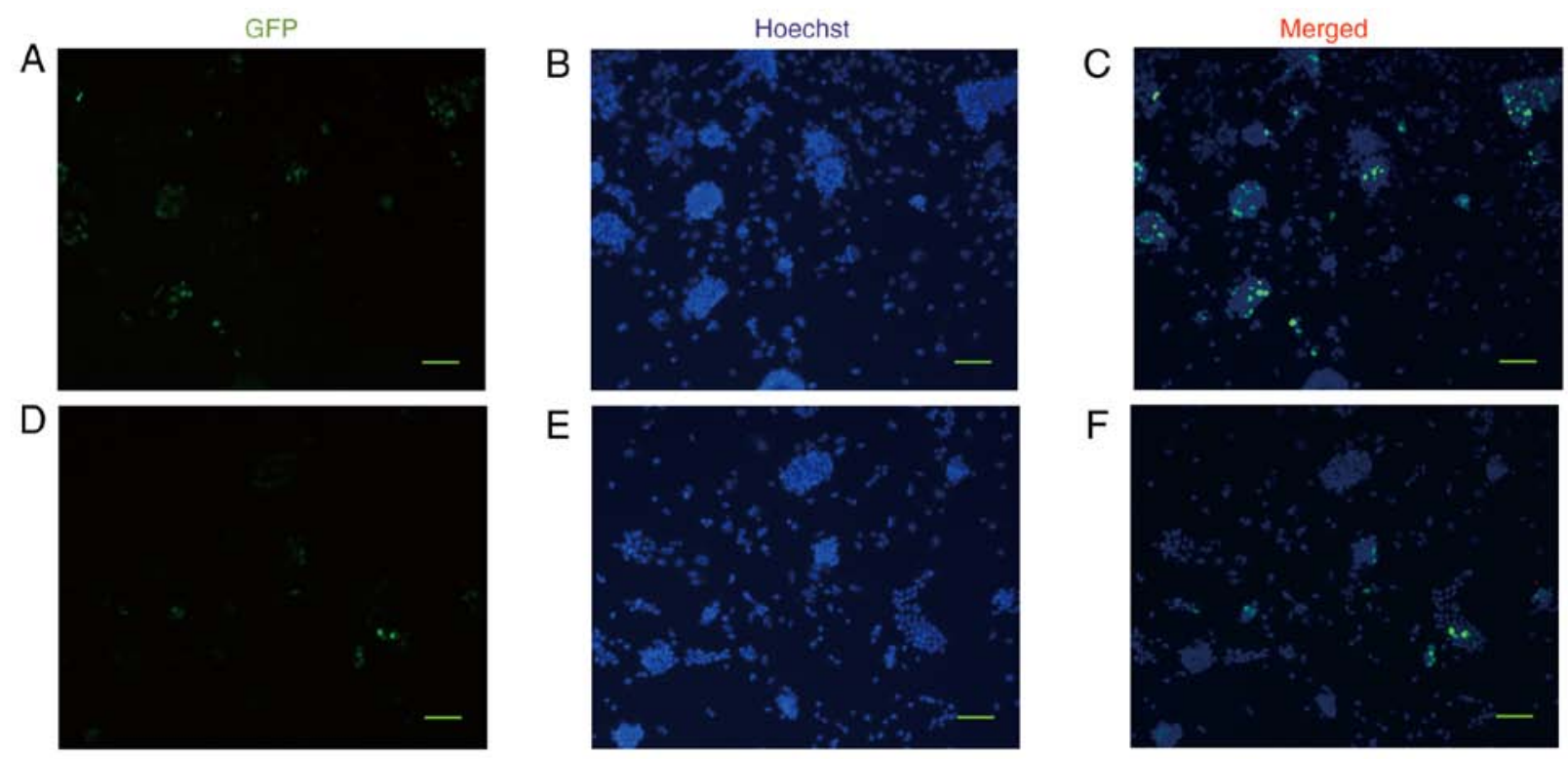

E

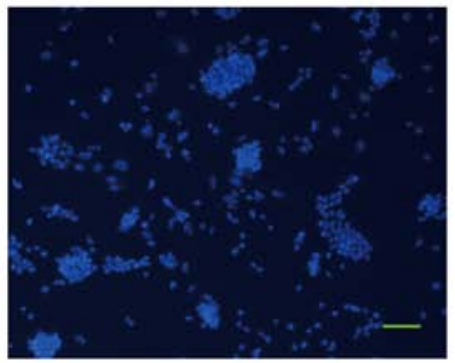

$\mathrm{H}$

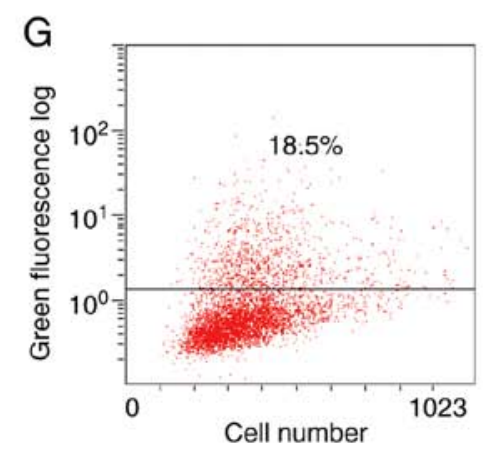

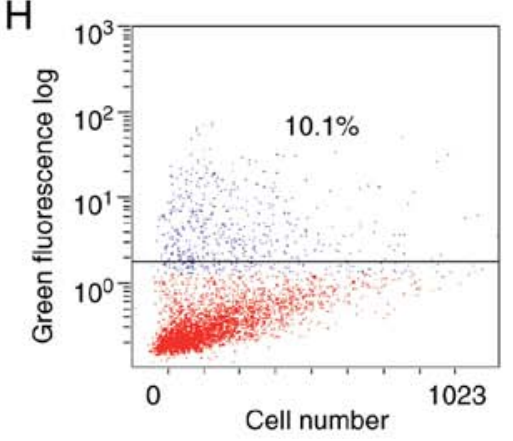

$\mathrm{F}$

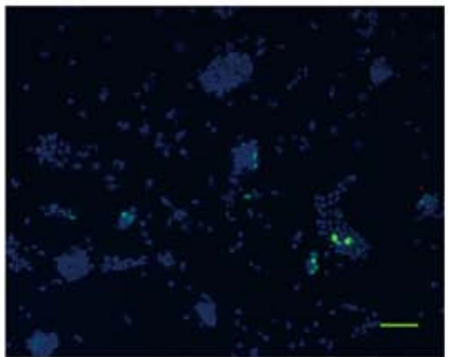

I

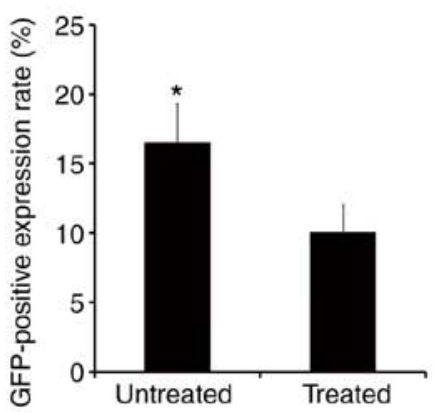

Figure 4. Transient transfection of the pMsil-GFP vector. pMsil-GFP was transfected into the 9-day cell population derived from mESCs without LY294002 treatment, as shown in (A) GFP, (B) Hoechst and (C) merged images, and with LY294002 treatment, as shown in (D) GFP, (E) Hoechst and (F) merged images. After $24 \mathrm{~h}$, GFP-positive cells were identified in both groups under a fluorescence microscope. Scale bar=50 $\mu \mathrm{m}$. At $24 \mathrm{~h}$ post-transfection with pMsi1-GFP, the positive expression rate of GFP in the 10-day cell population (G) without LY294002 treatment $(G)$ and $(H)$ with LY294002 treatment were calculated with flow cytometry. (I) Positive expression rate of GFP in the 10-day cell population without LY294002 treatment was higher than that in the 10-day cell population treated with LY294002. "P<0.05. Msi1, Musashi 1; mESCs, mouse embryonic stem cells; GFP, green fluorescent protein.

with the 10-day cell population without LY294002 (Fig. 6). The mRNA expression of Pdgfr- $\alpha$ in grafts from the 10-day cell population treated with LY294002 was higher than in the other groups $(\mathrm{P}<0.05)$. Therefore, LY294002 promoted the production of mesoderm during the process of mESC differentiation. The mRNA expression levels of Pdgfr- $\alpha$ in grafts from LY/EB-Msi1-positive cells were significantly lower than those from the 10-day cell populations treated with/without LY294002 $(\mathrm{P}<0.05)$. These results indicated that neither EB-Msi1-positive cells nor LY-Msil-positive cells had the tendency to develop into mesoderm tissue.

Immunohistochemical analysis of grafts. A higher number of Tubulin $\beta$ III-positive cells were detected in the grafts from the 10-day cell population without LY294002 and from EB-Msi1-positive cells than those from the 10-day cell population treated with LY294002 and LY-Msi1-positive cells (Fig. 7A-D). A high number of Villin-positive cells were detected in the grafts from the 10-day cell population treated with LY294002 and LY-Msi1-positive cells than in those from the 10-day cell population without LY294002 and EB-Msi1-positive cells (Fig. 7E-H).

\section{Discussion}

In our previous study, a pMsi1-GFP vector was successfully constructed, which was able to identify Msi1-positive cells, and to select Msi1-positive cells from a cell population originating from mESCs (16). Although the selected Msi1-positive cells had the potential to develop into neural and intestinal epithelial-like cells, the majority tended to develop into neural epithelial-like tissues. Only a small proportion of intestinal epithelial-like tissues were identified in the grafts from selected Msi1-positive cells. An additional way to harvest more intestinal epithelial-like tissues developed from Msi1-positive cells was to suppress the differentiation of mESCs into neutral epithelial-like tissue.

The ectoderm gives rise to the epidermis and neural epithelial tissue. Suppressing the differentiation of mESCs into ectoderm can prevent neutral epithelial tissue formation. The suppression of PI3K signaling efficiently promotes the differentiation of mESCs into mesendoderm and then definitive endoderm. It also prevents the differentiation of mESCs into ectoderm (27). PI3K signaling triggers the phosphorylation of GSK $3 \beta$ on Serine9, which can be inhibited with 

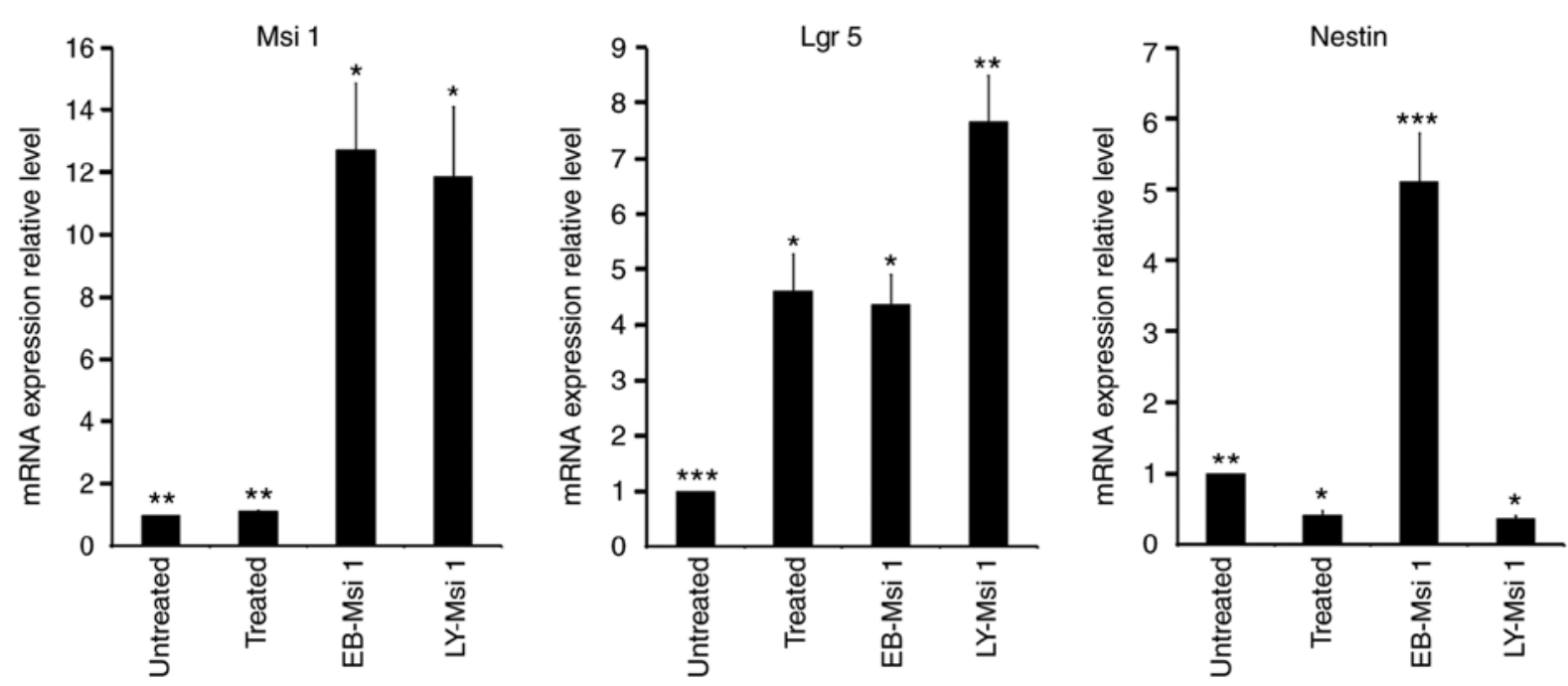

Figure 5. mRNA expression of symbols for neural and intestinal epithelial stem cells in selected Msi1-positive cells. The mRNA expression level of Msi1 in EB-Msi1 positive cells was similar to that in LY-Msi1-positive cells, and both were higher than that in the unselected 10-day cell population ("P $>0.05$; $\left.{ }^{* *} \mathrm{P}<0.05\right)$. The mRNA expression level of Lgr5 in EB-Msi1-positive cells was similar to that in the 10-day cell population treated with LY294002, and both were lower than that in LY-Msi1-positive cells but higher than that in the 10-day cell population without LY294002 treatment $\left({ }^{*} \mathrm{P}>0.05 ;{ }^{* * *} \mathrm{P}<0.05 ;{ }^{* * *} \mathrm{P}<0.05\right)$. The mRNA expression level of Nestin in LY-Msil-positive cells was similar to that in the 10-day cell population treated with LY294002, but both were significantly lower than that in the 10-day cell population without LY294002 treatment. The mRNA expression level of Nestin in LY-Msi1-positive cells was significantly lower than that in EB-Msil-positive cells $\left({ }^{*} \mathrm{P}>0.05 ;{ }^{* *} \mathrm{P}<0.05 ;{ }^{* * * *} \mathrm{P}<0.05\right)$. Msi1, Musashi 1; mESCs, mouse embryonic stem cells; EB, embryonic body; LY, LY294002; Lgr5, leucine-rich repeat-containing G-protein coupled receptor.
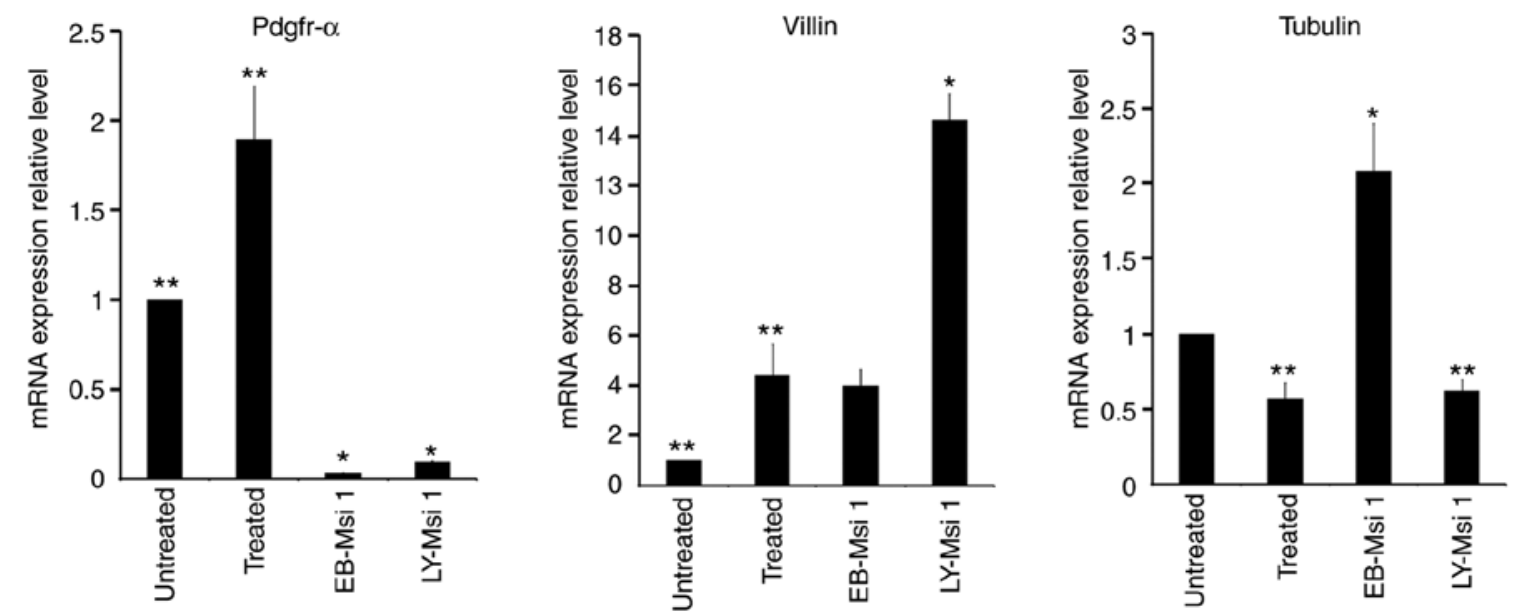

Figure 6. mRNA expression of markers for neural and intestinal epithelial cells in grafts. mRNA expression of Pdgfr- $\alpha$ in grafts from LY/EB-Msi1-positive cells were significantly lower than those from 10-day cell populations treated with/without LY294002 ("P<0.05). mRNA expression of Pdgfr- $\alpha$ in grafts from the 10-day cell population treated with LY294002 was significantly higher than that from 10-day cell population untreated without LY294002 treatment $\left({ }^{* *} \mathrm{P}<0.05\right)$. mRNA expression of Villin in grafts from LY-Msi1-positive cells was higher than in the other three groups $($ "P $<0.05)$. mRNA expression of Villin in grafts from the 10-day cell population treated with LY294002 was higher than that from the 10-day cell population without LY294002 treatment $\left.{ }^{* *} \mathrm{P}<0.05\right)$. mRNA expression of Tubulin $\beta$ III in grafts from EB-Msil-positive cells was higher than in the other three groups ("P<0.05). mRNA expression levels of Tubulin $\beta$ III in grafts from LY-Msi1-positive cells and the 10-day cell population treated with LY294002 were lower than that in the 10-day cell population without LY294002 treatment $\left({ }^{* *} \mathrm{P}<0.05\right)$. Msi1, Musashi 1; mESCs, mouse embryonic stem cells; EB, embryonic body; Pdgf- $\alpha$, platelet-derived growth factor receptor $\alpha$; LY, LY294002.

LY294002 (41). In the present study, LY294002 (5 $\mu \mathrm{mol} / \mathrm{l})$ effectively inhibited the activity of the PI3K signaling pathway, but had limited suppressive effect on the proliferation of mESCs. The concentration of DMSO used did not affect cell survival, which was consistent with the results of a study by Semba et al (42).

The PI3K signaling pathway can affect the differentiation of mESCs (43). The inhibition of PI3K signaling alters gene expression during the differentiation of mESCs (27). The
mRNA expression patterns of Msi1 in cells derived from mESCs treated with and without LY294002 were similar, however, the expression level in the treated group was lower than that in the untreated group during the differentiation of mESCs, with the exception of that in the 10-day cell population. The mRNA expression of Msil reached a peak level in the 11-day cell population derived from mESCs without LY294002 treatment, whereas the mRNA expression of Msi1 reached a peak level in the 10-day cell population derived 

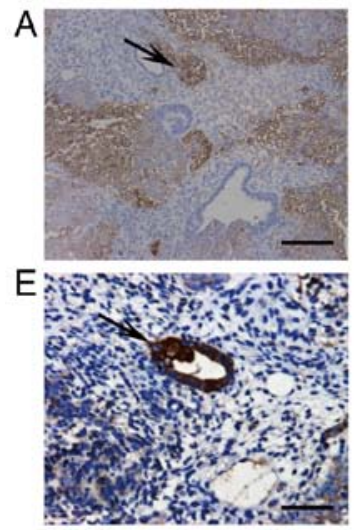
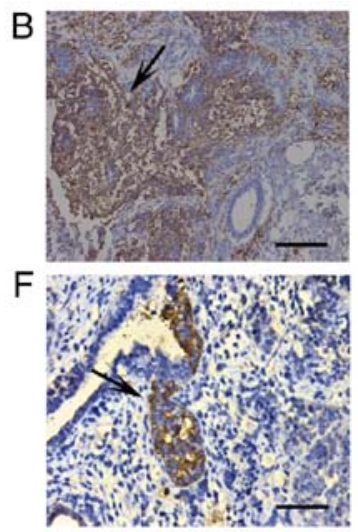
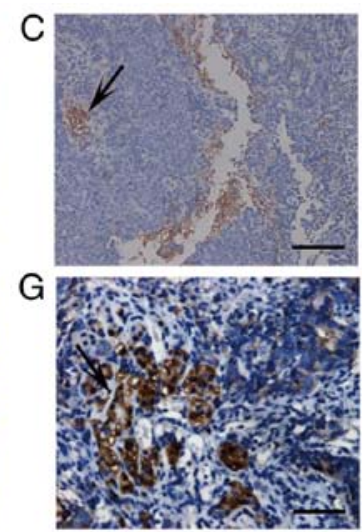
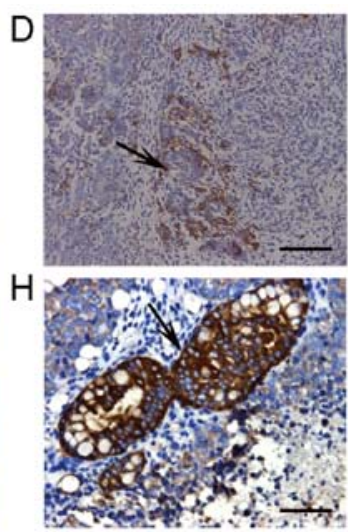

Figure 7. Immunohistochemical analysis of grafts. Tubulin $\beta$ III-positive cells (black arrow) were more prevalent in the grafts from the (A) 10-day cell population without LY294002 treatment and from (B) EB-Msil-positive cells than in the grafts from the (C) 10-day cell population treated with LY294002 and from (D) LY-Msi1-positive cells. Villin-positive cells (black arrow) were less prevalent in the grafts from the (E) 10-day cell population without LY294002 than in the grafts from the $(\mathrm{G}) 10$-day cell population treated with LY294002, and were less prevalent in the grafts from (F) EB-Msi1-positive cells than in the grafts from (H) LY-Msi1-positive cells. Scale bar=50 $\mu \mathrm{m}$. Msi1, Musashi 1; mESCs, mouse embryonic stem cells; EB, embryonic body.

from mESCs treated with LY294002. This result indicated that LY294002 accelerated the differentiation of mESCs, and shifted the peak mRNA expression of Msil to an earlier date. Following transient transfection with the pMsi1-GFP vector, fewer Msi1-positive cells were detected in the 10-day population derived from mESCs treated with LY294002 than in the untreated group. This result was inconsistent with the qPCR results, although the reason remains unclear. One possibility was that LY294002 may affect the expression of the pMsi1-GFP vector. As LY294002 suppressed the differentiation of mESCs into ectoderm, there was a corresponding reduction in the production of Msi1-positive cells, which are neuronal precursor cells $(6,15)$. Therefore, it was concluded that LY294002 did not increase the production of Msi1-positive cells derived from mESCs.

Although the mRNA expression of Msi1 was high in the EB-Msi-1- and LY-Msi1-positive cells, the mRNA expression levels of Lgr5 and Nestin were different. The mRNA expression level of Lgr5 in LY-Msi1-positive cells was higher than that in EB-Msil-positive cells and unselected cells derived from mESCs treated with/without LY294002, whereas the mRNA expression level of Nestin in EB-Msi1-positive cells was higher than that in LY-Msi1-positive cells and unselected cells derived from mESCs treated with/without LY294002. These results showed that LY-Msi1-positive cells appeared to contain more intestinal epithelial stem cells, whereas EB-Msi1-positive cells appeared to contain more neural epithelial stem cells. As Msi1 is considered a marker of neural and intestinal epithelial stem cells, it is reasonable to suggest that there two subsets of Msi1-positive cells were derived from mESCs; one subset was prone to differentiate into neural epithelial tissue, whereas the other was prone to differentiate into intestinal epithelial tissue (16). The suppression of PI3K signaling resulted in a reduction of the subset that differentiated into neural epithelial tissue and promoted the differentiation of mESCs into intestinal epithelial tissue.

As precursor cells of neural and intestinal epithelial cells, Msi1-positive cells lose the ability to differentiate into certain cell types, particularly mesodermal cells. The lower
mRNA expression level of Pdgfr- $\alpha$ in grafts derived from LY/EB-Msi1-positive cells provides direct evidence of this.

As mentioned above, the LY-Msi1-positive cells and EB-Msi1-positive cells had similar characteristics. As Msi1 is considered a candidate marker of intestinal epithelial stem cells, it is presumed that Villin-positive cells can be detected in the grafts from selected Msi1-positive cells $(9,37)$. There were also differences in the expression levels of markers of neural and intestinal epithelial cells. As shown in the results of the mRNA expression analysis and immunohistochemical analysis, the expression level of Villin was higher in grafts derived from cells treated with LY294002 than those from cells without LY294002 treatment. In addition, the expression level of Villin was higher in grafts derived from LY-Msi1-positive cells than in those from EB-Msi1-positive cells. These results revealed that LY294002 promoted the differentiation of mESCs into endoderm, and increased the production of intestinal epithelial-like cells. The Msi1-positive cells derived from mESCs treated with LY294002 were distinct from those without LY294002 treatment. Compared with EB-Msi1-positive cells, more LY-Msi1-positive cells differentiated into intestinal epithelial-like tissue. This result partially clarified the reason why LY294002 increased the production of intestinal epithelial-like tissue in the grafts derived from mESCs treated with LY294002.

The marker of neural epithelial cells, Tubulin $\beta$ III, was expressed at high levels in grafts derived from EB-Msi1-positive cells, whereas Villin, a marker of intestinal epithelial cells, was expressed at a lower level (35). This indicated that the majority of EB-Msi1-positive cells developed into neural epithelial-like tissue, and only a small number of EB-Msi1-positive cells differentiated into intestinal epithelial-like tissue (16). Therefore, EB-Msi1-positive cells can be regarded as 'neural epithelial stem cells', rather than 'intestinal epithelial stem cells'. As LY294002 inhibited the differentiation of mESCs into ectoderm, the expression levels of Tubulin $\beta$ III in grafts from unselected cells treated with LY294002 and LY-Msi1-positive cells were significantly lower than those from unselected cells without LY294002 treatment and EB-Msi1-positive cells, as shown in the results of the mRNA expression and 
immunohistochemical analyses. These results suggested that LY294002 inhibited the differentiation of mESCs into neural epithelial-like tissue (27).

In conclusion, the present study demonstrated that LY294002 promoted the differentiation of mESCs into intestinal epithelial-like tissue. The Msil-positive cells selected from a cell population derived from mESCs treated with LY294002 had more characteristics of intestinal epithelial stem cells, and were suitable for use as a platform for further differentiation. Due to the low proportion of Msi1-positive cells in mesendoderm cells, it is not possible to provide sufficient Msi1-positive cells for the investigation of intestinal epithelial stem cells. In order to sort out a higher number of Msi1-positive cells for intestinal epithelial stem cell research, further investigations are required focused on increasing the ratio of Msi1-positive cells in mesendoderm cells (44).

\section{Acknowledgements}

The authors are indebted to Dr. Xiao-xue Li for expertly reviewing of the manuscript.

\section{Funding}

The present study was supported by the Natural Science Foundation of Guangdong Province, China (grant no. 2014A030313396) and the Youth Innovative Talents Project of the Educational Department of Guangdong Province (grant no. 2016KQNCX028).

\section{Availability of data and materials}

All data generated or analyzed during this study are included in this published article.

\section{Authors' contributions}

SYL and MAT contributed equally to this work, and conducted the experiments and drafted the manuscript. SHY participated in the cell culturing. TY and JZC participated in the western blot analysis. BC and PWL participated in the reverse transcription-quantitative polymerase chain reaction studies. DMF and FBL performed the statistical analysis and helped to draft the manuscript. QKC participated in the design of the study and gave the final approval of the version to be published. All authors read and approved the final manuscript.

\section{Ethics approval and consent to participate}

All procedures were performed in accordance with the Animal Ethics Committee of the Second Affiliated Hospital of Sun Yat-Sen University.

\section{Patient consent for publication}

Not applicable.

\section{Competing interests}

The authors declare that they have no competing interests.

\section{References}

1. Nakamura M, Okano H, Blendy JA and Montell C: Musashi, a neural rna-binding protein required for drosophila adult external sensory organ development. Neuron 13: 67-81, 1994.

2. Okano H, Imai T and Okabe M: Musashi: A translational regulator of cell fate. J Cell Sci 115: 1355-1359, 2002

3. Sakakibara S, Imai T, Hamaguchi K, Okabe M, Aruga J, Nakajima K, Yasutomi D, Nagata T, Kurihara Y, Uesugi S, et al: Mouse-Musashi-1, a neural RNA-Binding protein highly enriched in the mammalian CNS stem cell. Dev Biol 176: 230-242, 1996.

4. Potten CS, Booth C, Tudor GL, Booth D, Brady G, Hurley P, Ashton G, Clarke R, Sakakibara S and Okano H: Identification of a putative intestinal stem cell and early lineage marker; Musashi-1. Differentiation 71: 28-41, 2003.

5. Okano H, Kawahara H, Toriya M, Nakao K, Shibata S and Imai T: Function of RNA-binding protein Musashi-1 in stem cells. Exp Cell Res 306: 349-356, 2005.

6. Sakatani T, Kaneda A, Iacobuzio-Donahue CA, Carter MG, de Boom Witzel S, Okano H, Ko MS, Ohlsson R, Longo DL and Feinberg AP: Loss of imprinting of Igf2 alters intestinal maturation and tumorigenesis in mice. Science 307: 1976-1978, 2005.

7. Arumugam K, Macnicol MC and Macnicol AM: Autoregulation of Musashil mRNA translation during Xenopus oocyte maturation. Mol Reprod Dev 79: 553-563, 2012.

8. Booth C and Potten CS: Gut instincts: Thoughts on intestinal epithelial stem cells. J Clin Invest 105: 1493-1499, 2000.

9. Kayahara T, Sawada M, Takaishi S, Fukui H, Seno H, Fukuzawa H, Suzuki K, Hiai H, Kageyama R, Okano H and Chiba T: Candidate markers for stem and early progenitor cells, Musashi-1 and Hes1, are expressed in crypt base columnar cells of mouse small intestine. FEBS Lett 535: 131-135, 2003.

10. Fukui T, Takeda H, Shu HJ, Ishihama K, Otake S, Suzuki Y, Nishise S, Ito N, Sato T, Togashi H and Kawata S: Investigation of Musashi-1 expressing cells in the murine model of dextran sodium sulfate-induced colitis. Dig Dis Sci 51: 1260-1268, 2006.

11. Kaneko $\mathrm{J}$ and Chiba $\mathrm{C}$ : Immunohistochemical analysis of Musashi-1 expression during retinal regeneration of adult newt. Neurosci Lett 450: 252-257, 2008.

12. Yamada T, Yoshikawa M, Takaki M, Torihashi S, Kato Y, Nakajima Y, Ishizaka S and Tsunoda Y: In vitro functional gut-like organ formation from mouse embryonic stem cells. Stem Cells 20: 41-49, 2002.

13. Takaki M, Nakayama S, Misawa H, Nakagawa T and Kuniyasu H: In vitro formation of enteric neural network structure in a gut-like organ differentiated from mouse embryonic stem cells. Stem Cells 24: 1414-1422, 2006.

14. Kaneko Y, Sakakibara S, Imai T, Suzuki A, Nakamura Y Sawamoto K, Ogawa Y, Toyama Y, Miyata T and Okano H: Musashil: An evolutionally conserved marker for CNS progenitor cells including neural stem cells. Dev Neurosci 22: 139-153, 2000.

15. Maslov AY, Barone TA, Plunkett RJ and Pruitt SC: Neural stem cell detection, characterization, and age-related changes in the subventricular zone of mice. J Neurosci 24: 1726-1733, 2004.

16. Lan SY, Yu T, Xia ZS, Yuan YH, Shi L, Lin Y, Huang KH and Chen QK: Musashi 1-positive cells derived from mouse embryonic stem cells can differentiate into neural and intestinal epithelial-like cells in vivo. Cell Biol Int 34: 1171-1180, 2010.

17. Simon TC and Gordon JI: Intestinal epithelial cell differentiation: New insights from mice, flies and nematodes. Curr Opin Genet Dev 5: 577-586, 1995.

18. Hellmich HL, Kos L, Cho ES, Mahon KA and Zimmer A: Embryonic expression of glial cell-line derived neurotrophic factor (GDNF) suggests multiple developmental roles in neural differentiation and epithelial-mesenchymal interactions. Mech Dev 54: 95-105, 1996.

19. Gao N, White P and Kaestner KH: Establishment of intestinal identity and epithelial-mesenchymal signaling by. Dev Cell 16: 588-599, 2009.

20. Pai YJ, Abdullah NL, Mohd-Zin SW, Mohammed RS, Rolo A Greene ND, Abdul-Aziz NM and Copp AJ: Epithelial fusion during neural tube morphogenesis. Birth Defects Res A Clin Mol Teratol 94: 817-823, 2012.

21. Zheng H, Fu G, Dai T and Huang H: Migration of endothelial progenitor cells mediated by stromal cell-derived factor-1alpha/CXCR4 via PI3K/Akt/eNOS signal transduction pathway. J Cardiovasc Pharmacol 50: 274-280, 2007. 
22. Yu J, Li M, Qu Z, Yan D, Li D and Ruan Q: SDF-1/CXCR4mediated migration of transplanted bone marrow stromal cells toward areas of heart myocardial infarction through activation of PI3K/Akt. J Cardiovasc Pharmacol 55: 496-505, 2010.

23. Wang H, Yin Y, Li W, Zhao X, Yu Y, Zhu J, Qin Z, Wang Q, Wang $\mathrm{K}, \mathrm{Lu} \mathrm{W}$, et al: Over-expression of PDGFR- $\beta$ promotes PDGF-induced proliferation, migration, and angiogenesis of EPCs through PI3K/Akt signaling pathway. PLoS One 7: e30503, 2012.

24. Cantley LC: The phosphoinositide 3-kinase pathway. Science 296: 1655-1657, 2002.

25. Paling NR, Wheadon H, Bone HK and Welham MJ: Regulation of embryonic stem cell self-renewal by phosphoinositide 3-kinase-dependent signaling. J Biol Chem 279: 48063-48070, 2004.

26. Storm MP, Kumpfmueller B, Thompson B, Kolde R, Vilo J, Hummel O, Schulz $\mathrm{H}$ and Welham MJ: Characterization of the phosphoinositide 3-kinase-dependent transcriptome in murine embryonic stem cells: Identification of novel regulators of pluripotency. Stem Cells 27: 764-775, 2009.

27. Mclean AB, D'Amour KA, Jones KL, Krishnamoorthy M, Kulik MJ, Reynolds DM, Sheppard AM, Liu H, Xu Y, Baetge EE and Dalton S: Activin a efficiently specifies definitive endoderm from human embryonic stem cells only when phosphatidylinositol 3-kinase signaling is suppressed. Stem Cells 25: 29-38, 2007.

28. Roche S, Koegl M and Courtneidge SA: The phosphatidylinositol 3-kinase alpha is required for DNA synthesis induced by some, but not all, growth factors. Proc Natl Acad Sci USA 91: 9185-9189, 1994

29. Shivakrupa R, Bernstein A, Watring $N$ and Linnekin D: Phosphatidylinositol 3'-kinase is required for growth of mast cells expressing the kit catalytic domain mutant. Cancer Res 63 : 4412-4419, 2003.

30. Livak KJ and Schmittgen TD: Analysis of relative gene expression data using real-time quantitative PCR and the 2(T)(-Delta Delta C) method. Methods 25: 402-408, 2001.

31. Lianguzova MS, Chuykin IA, Nordheim A and Pospelov VA: Phosphoinositide 3-kinase inhibitor LY294002 but not serum withdrawal suppresses proliferation of murine embryonic stem cells. Cell Biol Int 31: 330-337, 2007.

32. May R, Sureban SM, Hoang N, Riehl TE, Lightfoot SA, RamanujamR,WycheJH,AnantS andHouchenCW:Doublecortin and cam kinase-like-1 and leucine-rich-repeat-containing G-protein-coupled receptor mark quiescent and cycling intestinal stem cells, respectively. Stem Cells 27: 2571-2579, 2009.

33. Braun H, Schäfer K and Höllt V: BetaIII tubulin-expressing neurons reveal enhanced neurogenesis in hippocampal and cortical structures after a contusion trauma in rats. J Neurotrauma 19: 975-983, 2002
34. Guo J, Walss-Bass C and Ludueña RF: The beta isotypes of tubulin in neuronal differentiation. Cytoskeleton (Hoboken) 67: 431-441, 2010.

35. Guo J, Qiang M and Ludueña RF: The distribution of $\beta$-tubulin isotypes in cultured neurons from embryonic, newborn, and adult mouse brains. Brain Res 1420: 8-18, 2011.

36. Maunoury R, Robine S, Pringault E, Huet C, Guénet JL, Gaillard JA and Louvard D: Villin expression in the visceral endoderm and in the gut anlage during early mouse embryogenesis. EMBO J 7: 3321-3329, 1988.

37. Pinto D, Robine S, Jaisser F, EI Marjou FE and Louvard D: Regulatory sequences of the mouse villin gene that efficiently drive transgenic expression in immature and differentiated epithelial cells of small and large intestines. J Biol Chem 274: 6476-6482, 1999.

38. Takakura N, Yoshida H, Ogura Y, Kataoka H, Nishikawa S and Nishikawa S: PDGFR alpha expression during mouse embryogenesis: Immunolocalization analyzed by whole-mount immunohistostaining using the monoclonal anti-mouse PDGFR alpha antibody APA 5. J Histochem Cytochem 45: 883-893, 1997.

39. Karlsson L, Lindahl P, Heath JK and Betsholtz C: Abnormal gastrointestinal development in PDGF-A and PDGFR-(alpha) deficient mice implicates a novel mesenchymal structure with putative instructive properties in villus morphogenesis. Development 127: 3457-3466, 2000.

40. Takebe A, Era T, Okada M, Martin Jakt L, Kuroda Y and Nishikawa S: Microarray analysis of PDGFR $\alpha+$ populations in ES cell differentiation culture identifies genes involved in differentiation of mesoderm and mesenchyme including ARID3b that is essential for development of embryonic mesenchymal cells. Dev Biol 293: 25-37, 2006.

41. Oviedo-Boyso J, Cortés-Vieyra R, Huante-Mendoza A, Yu HB, Valdez-Alarcón JJ, Bravo-Patiño A, Cajero-Juárez M, Finlay BB and Baizabal-Aguirre VM: The phosphoinositide-3-kinase-Akt signaling pathway is important for Staphylococcus aureus internalization by endothelial cells. Infect Immun 79: 4569-4577, 2011.

42. Semba $S$, Itoh $N$, Ito $M$, Harada $M$ and Yamakawa $M$ : The in vitro and in vivo effects of 2-(4-morpholinyl)-8-phenyl-chromone (LY294002), a specific inhibitor of phosphatidylinositol 3'-kinase, in human colon cancer cells. Clin Cancer Res 8: 1957-1963, 2002.

43. Xu XY, Zhang Z, Su WH, Zhang Y, Feng C, Zhao HM, Zong ZH, Cui C and Yu BZ: Involvement of the p110 alpha isoform of PI3K in early development of mouse embryos. Mol Reprod Dev 76: 389-398, 2009.

44. van der Flier LG and Clevers H: Stem cells, self-renewal, and differentiation in the intestinal epithelium. Annu Rev Physiol 71: 241-260, 2009. 